\title{
Los videos educativos como herramienta disruptiva para apoyar el proceso de aprendizaje de algoritmos de resta y multiplicación en estudiantes de segundo grado de primaria
}

Velasco Guardias, Angélica María; Montiel Bautista, Susana; Ramírez García, Susana

Los videos educativos como herramienta disruptiva para apoyar el proceso de aprendizaje de algoritmos de resta y multiplicación en estudiantes de segundo grado de primaria

Revista Educación, vol. 42, núm. 2, 2018

Universidad de Costa Rica, Costa Rica

Disponible en: http://www.redalyc.org/articulo.oa?id=44055139009

DOI: https://doi.org/10.15517/revedu.v42i2.24236

Esta obra está bajo una Licencia Creative Commons Atribución-NoComercial-SinDerivar 3.0 Internacional. 


\title{
Los videos educativos como herramienta disruptiva para apoyar el proceso de aprendizaje de algoritmos de resta y multiplicación en estudiantes de segundo grado de primaria
}

\author{
Educational Videos as a Disruptive Tool to Support the Learning Process of Subtraction and Multiplication \\ Algorithms in Second Grade Primary School Children
}

Angélica María Velasco Guardias [1]

Fundación Gimnasio Moderno, Colombia

vangelg7737@gmail.com

Susana Montiel Bautista [2]

Tecnológico de Monterrey, México

susanamontiel@tecvirtual.mx

Susana Ramirez Garcia [3]

Tecnológico de Monterrey, México

sramirezg@itesm.mx

\section{RESUMEN:}

La presente investigación fue realizada en una institución de estudiantes varones para fortalecer la enseñanza de las matemáticas, el tema que se seleccionó fue la competencia de desarrollo de algoritmos de resta y multiplicación por una cifra, para niños de grado segundo, cuyas edades oscilan entre 8 y 9 años, como muestra de estudio se tomaron 24 estudiantes 5 con dificultades de aprendizaje. Es una investigación cuantitativa de tipo experimental. Los instrumentos que se emplearon fueron la observación, dos test de habilidades específicas y una entrevista, los cuales se analizaron desde dos categorías y fueron la base para la investigación:

\section{NotAS DE AUTOR}

[1] Licenciada en Matemáticas de la Universidad Pedagogica Nacional y Maestría enTecnologia Educativa y Medios Innovadores para la Educación de la UNAB y el Tecnologico de Monterrey. La experiencia laboral de la licenciada Angélica comenzo en un colegio oficial de la ciudad de Bogotá, Colombia, como docente de matemáticas de Bachillerato, año y medio despues empezó a trabajar en un colegio privado de la población de Cajica, luego de año y medio inició su trabajo en la Fundación Gimnasio Moderno, lugar donde labora hasta la actualidad y se desempeña como dicente de matematicas con niñez de segundo y tercer grado de primaria. Dentro de su trabajo de maestria realizó la publicación de un articulo para la revista TE\&ET de Argentina, titulado "El mundo de las fracciones: Blog interactivo que favorece la resolución de problemas con fracciones en alumnos de nivel educativo básico y medio", este trabajo se realizo en compañía de otras compañeras de estudio.

[2] Licenciada en Educación Media en la Especialidad de Español y Maestra en Tecnología Educativa con acentuación en medios innovadores para la educación por el ITESM. La experiencia profesional de maestra Susana Montiel Bautista comenzó en la iniciativa privada, trabajando para la empresa Coca-Cola, su experiencia en los últimos 16 años ha girado principalmente en el campo educativo, como profesora en educación básica. Ha impartido cursos de habilidades docentes a profesorado de nivel licenciatura, ha participado en diplomados en línea dirigidos a docentes, se desempeña también como tallerista con temas como el constructivismo y la importancia de la recuperación de los conocimientos previos, planificación docente, preguntas generadoras, entre otros. Tiene especial interés en lograr aprendizajes relevantes en estudiantes, motivándoles en el desarrollo de habilidades de pensamiento, considera que las tecnologías de la información y la comunicación deben aprovecharse al máximo tanto en el contexto escolar como fuera de este. Actualmente es docente en nivel secundaria, de la asignatura de Español; forma parte de la Cátedra de investigación del ITESM, se desempeña en la Escuela de Humanidades y Ciencias Sociales en el Instituto Tecnológico y de Estudios Superiores de Monterrey como profesora tutora de proyectos de investigación aplicada.

[3] Doctora en Educación por la Universidad Autónoma de Nuevo León, con mención honorifica de Cum Laude en tesis publicada con arbitraje estricto y de circulación internacional. Con Maestría en Psicología Educativa por la Universidad Regiomontana. Es licenciada en Psicología con acentuación en Psicología Social por la Universidad Autónoma de Nuevo León. Su experiencia profesional reside desde 1994 en el ámbito psicológico y educativo, en sectores públicos y privados. Se ha desempeñado como asesora educativa (profesora tutora) y profesor titular en los programas y tesis de posgrado en Escuela de Graduados en Educación del Sistema Tecnológico de Monterrey Actualmente es profesora titular de proyectos de investigación en la MEE y MTE. Ha participado en diversos congresos nacionales e internacionales como ponente en diversos temas y publicaciones sobre educación, tecnología, salud y psicología. Ha sido coordinadora de cambios curriculares y plan de estudios en nivel superior y diseñadora instruccional en proyectos y cursos empresariales, académicos y de capacitación y evaluación del ITESM. Del año 1997 a la fecha, es docente en licenciatura y posgrado en la Universidad Regiomontana en la Facultad de Humanidades y Ciencias Sociales. Se ha desempeñado en diversas áreas de la psicología como la clínica, hospitalaria, instituciones infantiles y educativas. Ha sido coordinadora del área de psicología de la estancia infantil. ISSSTE. Estancia de Bienestar y Desarrollo Infantil de Oaxaca. (1996) Fue docente de la licenciatura en área básica y de especialidad en psicología en la Universidad Autónoma de Nuevo León. 
los conceptos trabajados y el uso del video educativo. La pregunta de investigación fue: ¿Qué efectos se evidencian al implementar videos educativos como herramienta disruptiva para apoyar el proceso de aprendizaje de restas y multiplicaciones en los estudiantes de segundo primaria? El objetivo de la investigación era implementar el uso de videos tutoriales como recursos educativos para fortalecer el proceso de enseñanza de restas y multiplicaciones en la población infantil de segundo primaria con dificultades de aprendizaje y sin estas. Los efectos evidenciados con el uso de los videos fueron: incremento en los periodos de atención y concentración, más confianza en la ejecución de nuevos ejercicios, el crecimiento de las habilidades se vio directamente relacionada con la cantidad de veces que se vieron los videos. Los principales hallazgos fueron: la población con trastorno de déficit de atención (TDAH) y dislexia que puede estar acompañada de discalculia se vio beneficiada al observar los videos; en cuanto a la que no presenta dificultades de aprendizaje se determinó que los emplearon como fuente de estudio y consulta. Este proyecto demuestra que el uso de los videos en la educación ayuda a niños y niñas con dificultades cognitivas y apoya el proceso de estudio de quienes no presentan dificultades. Los videos empleados son de propia autoría y todos se encuentran de forma libre en YouTube.

Palabras Clave: Videos educativos, discalculia, disrupción, aprendizaje.

\section{Abstract:}

The present investigation was carried out to strengthen the teaching of mathematics. The topic selected was the competence to develop one digit subtraction and multiplication algorithms for second grade children, whose ages range between 8 and 9 years. As a sample of study, 24 children were chosen, 5 out the total with learning difficulties. It is a quantitative research with an experimental design. The instruments used were: observation, two tests of specific skills and an interview analyzed under two categories. The concepts and the use of educational videos worked as the research base. The research question was: what effects are evidenced when implementing educational videos as a disruptive tool to support the process of subtraction and multiplication learning in children in second grade? The objective of the research was to: implement the use of tutorial videos as an educational resource to strengthen the process of teaching subtraction and multiplication in second grade children with and without learning difficulties. The evidence-based effects of the use of videos were: increase in the periods of attention and concentration, more confidence in the execution of new exercises, the development of the skills was directly related to the number of times the videos were viewed. The main findings were: children with attention deficit disorder (ADD) and dyslexia that may be accompanied by dyscalculia benefited from watching the videos. Regarding children who do not have learning difficulties, it was determined that they used them as a source of study and consultation. This project demonstrates that the use of videos in education helps children with cognitive difficulties and supports the study process of students who do not have learning difficulties. The videos used are of own authorship and are available on YouTube.

KEYWORDS: educational videos, dyscalculia, disruption, learning.

\section{INTRODUCCIÓN}

En este artículo se utilizaron los videos educativos creados desde las necesidades vistas en niños, todos de autoría propia, como una herramienta para apoyar el aprendizaje de las matemáticas en segundo grado. Puntualmente se estudió la solución de algoritmos correspondientes a la sustracción y la multiplicación por una cifra. Se trabajó en un colegio varones de Bogotá, Colombia, el grupo de estudio fue de 24 estudiantes, 5 con dificultades de aprendizaje. Este es un colegio con un modelo educativo de escuela activa, en el cual se permite la libertad de cátedra y el uso de tecnologías, sin embargo, no se emplean y el modelo se convierte en tradicional. En el marco conceptual se hace referencia a la discalculia y al déficit de atención como algunas dificultades de aprendizaje que presentan niños y niñas de cualquier institución educativa. La investigación se realizó con un método de investigación cuantitativa de carácter experimental, teniendo como grupo de control a la muestra anteriormente mencionada. Se establecieron dos ejes temáticos para la organización de datos: en primer lugar, los conceptos trabajados y en segundo, el uso de los vídeos educativos.

Para determinar los beneficios de la investigación, se emplearon tres herramientas, estas fueron: dos test, uno aplicado antes de ver los vídeos, llamado también pretest y en un segundo momento, después de visualizarlos o postest, otra herramienta utilizada fue la observación de actitudes y finalmente una entrevista que se les hizo a la población infantil después de terminar todo el proceso.

Con estas herramientas, y teniendo en cuenta los dos ejes de estudio, se realizó el análisis de los datos y se determinó la respuesta a la pregunta de investigación y el alcance del objetivo de esta misma. 
$\mathrm{Al}$ analizar los resultados obtenidos se pudo determinar que este tipo de visualizaciones aumentaron los tiempos de atención, el dominio de los algoritmos y a mayor número de veces que veían los videos, mayor era su confianza al afrontar tareas semejantes.

\section{Antecedentes prácticos y uso de videos en la educación}

El apoyo audiovisual se ha convertido en una fuerte herramienta en la enseñanza de varias asignaturas y en todos los niveles académicos. En Colombia, una universidad desarrolló un proyecto empleando tecnología educativa en cursos de gerontología, dicho proyecto consistía en emplear únicamente videos educativos e informativos para generar en sus estudiantes los conocimientos en esta área de estudio (Peña, García y Ramírez 2010).

En la Universidad de Alicante en España, fueron incorporados videos como evidencia empírica en el proceso del aprendizaje de matemáticas en niñez de primaria, y para la formación de docentes en la universidad. Lo primero que se hizo en la investigación fue grabar a los grupos mientras desarrollaban ejercicios matemáticos, luego empleando el estudio de casos se analizaron los videos y con base en estos se realizaron cambios curriculares y estrategias didácticas de trabajo con estudiantes (Llinares, 2004). En este caso los videos fueron creados para ver las debilidades del grupo, más no para solucionarlas.

El profesor de matemáticas José Andalón, en el año 2009, tomo la determinación de crear una maratón matemático, debido a que gran cantidad de sus estudiantes en México vivian muy lejos, empezó a crear videos y mostrarlos empleando internet, su compañera María Sánchez se une a ese trabajo y en conjunto crearon, en el 2011, un canal en YouTube llamado Math2me. La página tiene más de 143,758 visitas. La cantidad de visitas y comentarios que tiene esta página demuestran los grandes resultados de este proyecto. En lugares como Tijuana y el Estado de Guerrero quienes no tienen acceso a internet pueden ver por televisión los videos educativos (Alfaro, Monroy y Pinzón, 2013).

Salman Khan, en el año 2004, quería ayudar a una sobrina, que no vivía cerca de él, con una tarea, por esta razón creo su primer video tutorial, poco a poco fue creando más videos y subiéndolos a YouTube, hasta llegar a crear una gran plataforma de aprendizaje llamada Khan Academy, en la cual se encuentran videos y otras herramientas didácticas en su mayoría en inglés en las áreas de matemáticas, ciencia, historia, economía entre otras. Esta plataforma es gratuita y con el empleo de avatares va mostrando el avance de cada estudiante ya sea de forma autónoma o con la asesoría de docentes, es una plataforma abierta para niños, niñas, maestras y maestros (Rodríguez, 2011).

Los videos ayudan a consolidar conocimientos gracias a los estímulos audiovisuales y auditivos que contiene, además de ser importante en disminuir la brecha digital que existe entre el mundo de la escuela y el mundo alrededor de la infancia (Greenberg y Zanetis, 2012). Al estar en la web, el estudiantado puede reproducirlos cuantas veces desee sin ninguna restricción.

\section{MARCO TEÓRICO}

Este apartado está enfocado en mostrar cómo, con el uso de videos educativos en matemáticas, se puede apoyar el proceso de aprendizaje de la niñez, además el porqué son importantes las TIC en la educación, partiendo desde su definición hasta su implementación. Al final se describen algunas investigaciones relacionadas con la temática motivo de estudio que se han desarrollado sobre las tecnologías disruptivas en la educación, en especial, en proyectos enfocados en el uso de videos educativos. 


\section{Qué son las TIC y su importancia en la educación}

La sigla TIC, significa tecnologías de la información y la comunicación. Un estudio, realizado en el año 2007, establece que no es posible dar una definición única de lo que son las TIC ya que cuantas más fuentes se investigan, más diversas son las definiciones que se pueden encontrar (Cobo, 2009). "Las tecnologías de la información y de la comunicación (TIC) son la palanca principal de transformaciones sin precedentes en el mundo contemporáneo" (Carneiro, Toscano y Díaz, 2009).

Según la ley N.o1341 del 30 de Julio del 2009 de la República de Colombia, las TIC se definen como el conjunto de elementos tecnológicos, ya sean electrónicos como computadores, tabletas, ipads, entre otros; programas tecnológicos o redes y todas las conexiones que estas generan. Desde el año 2006, en todos los colegios públicos distritales (Bogotá), se realizó una gran dotación de equipos, programas y redes de internet, que están enfocadas en mejorar la calidad de la educación y la implementación del uso de las TIC en cualquier nivel económico en la capital. Para la clase de matemáticas estos colegios contaban con el paquete de Cabri Geometry Plus y Derive, esto se hace evidente al trabajar en diferentes colegios distritales y ver que todos tenían las mismas herramientas. Desde hace 10 años, se ha implementado en los colegios el uso de las TIC en todas las áreas del conocimiento, porque antes del 2004 solo se empleaban en la asignatura denominada informática. Tras el rápido avance tecnológico y las facilidades para la adquisición de equipos y software especializados, mayor número de instituciones han ampliado la cantidad de herramientas tecnológicas en sus aulas. Así mismo se ha implementado en Colombia capacitación a docentes que trabajan en el sector público, y en los colegios privados es su personal directivo el encargado de que sean competentes en el ámbito tecnológico (Plan decenal de educación 2006 - 2016).

Esta nueva era es de estudiantes de una generación digital (Rubio 2010). Al involucrarse todo el tiempo con nuevas tecnologías, sus procesos de aprendizaje están cada vez más ligados a este tipo de entornos. Según Sousa (2006), el cerebro aprende según la motivación y lo significativo que sean las experiencias de adquisición del conocimiento, cuanto más valor le puedan dar a las experiencias vividas se puede garantizar una mayor apropiación del conocimiento, y es en ese momento cuando se puede decir que se cumplió con el objetivo de la educación.

Las TIC brindan a jóvenes experiencias que antes eran casi imposibles de tener, ahora se pueden hacer laboratorios de química sin peligro de accidentes, modelar procesos físicos que no son fáciles de ver en la vida diaria, crear espacios que logran llevar a toda la juventud en niveles más similares de forma conceptual (Rubio, 2010).

Las nuevas tecnologías y el uso de estas han empezado a cambiar el modo de enseñanza - aprendizaje, de cómo este proceso se centra completamente en el estudiantado y en su deseo de aprender, al generar autocontrol y autonomía (Burbules, 2012). La importancia que tienen los maestros y maestras en el proceso de aprendizaje, depende directamente del modelo pedagógico que emplean en sus clases; en un modelo ubicuo, que esté en constante cambio y renovación, es indispensable que el estudiantado tenga la necesidad de aprender. Enseñar de esta forma ayuda a conectar a sus estudiantes con el mundo real y la aplicación de los conocimientos, pero a su vez implica para cada docente conocer ampliamente las herramientas que va a emplear y el cómo implementarlas para que sean efectivas en el aula. En este sentido, es necesario que el personal docente cuente con la capacitación y el tiempo necesario para el diseño y ejecución de proyectos exitosos que involucren nuevas tecnologías.

\section{Investigaciones acerca de tecnologías disruptivas}

A continuación se describen algunas investigaciones que se han desarrollado a nivel educativo empleando tecnologías que de alguna forma generan disrupción en el aula. 
En Brasil, la Universidad de la Sabana creó una materia electiva con la finalidad de que docentes de cualquier nivel de educación aprendan a manejar diferentes tecnologías emergentes, que ayuden a sus estudiantes a crear nuevos conceptos en diferentes espacios. En esta electiva cada docente debía seleccionar una herramienta tecnológica, definirla, trabajar con ella, diseñar proyectos, y determinar si su uso es o no apropiado en la educación. Las categorías del estudio se basaron en la aplicación que cada maestro o maestra daba a su herramienta tecnológica en sus respectivos espacios de trabajo. Como resultado de ese curso, aprendieron a diferenciar las herramientas útiles y las que no lo son, según su área de conocimiento (Boude, 2013).

En Colombia, por el proyecto uso y apropiación de las TIC, durante el gobierno del presidente Juan Manuel Santos, el Ministerio de Cultura de Colombia tuvo un aporte de U\$3.2 M, donado por la Fundación Gates. Con este dinero se realizó la primera fase de un proyecto que pretendía que se ampliara el uso y la apropiación de las TIC en 26 bibliotecas públicas ubicadas en diferentes partes del país, para esto se realizó un trabajo de diagnóstico, con el fin de determinar las necesidades reales de los lugares seleccionados; en este estudio se tuvo en cuenta la infraestructura física y tecnológica de todas las bibliotecas públicas nacionales, además se determinaron las necesidades de información que tenía el público usuario de cada biblioteca, con base en este estudio, se diseñó una experiencia piloto. Debido a este estudio, la Fundación Bill \& Melinda Gates aprobó un nuevo aporte de U\$15 M, para iniciar la segunda fase del Proyecto. Esta se llevó a cabo desde mayo de 2014 hasta marzo de 2018 (Ministerio de Cultura, 2014).

\section{Dificultades en el aprendizaje de las matemáticas}

Existen factores de riesgo en el aprendizaje de las matemáticas, tales como: las constitucionales, que tienen que ver con las condiciones físicas del estudiantado; las familiares, en las cuales se hace alusión a la pobreza y malas condiciones en el hogar; las emocionales e interpersonales, en estas se encuentran las condiciones afectuosas del estudiantado; las intelectuales y académicas, son quienes tienen trastornos de aprendizaje; los ecológicos, que se relacionan a los vecindarios peligrosos y, por último, se nombran los acontecimientos de la vida que generan estrés, en esta categoría entran las pérdidas de familiares cercanos y queridos (Aranda, Pérez y Sánchez, 2009).

Este proyecto se enfoca en las condiciones relacionadas con los riesgos intelectuales y académicos, ya que los otros factores no corresponden al contexto escolar de la población infantil con la cual se desarrolló el trabajo.

Con el avance de la ciencia, se ha descubierto que algunos niños y niñas presentan problemas con las bases matemáticas, debido a un trastorno del aprendizaje conocido con el nombre de discalculia, la cual impide que desarrollen competencias propias de las matemáticas tales como el conteo, el manejo y reconocimiento de los números, manejo y reconocimiento espacial y, por tanto, los procesos más complejos (Rosselli y Matute, 2011). Para tratar esta enfermedad se recomienda basarse en el diagnóstico neurológico y emplear juegos y herramientas tecnológicas diseñadas para la reeducación (Serra, 2015).

La discalculia está muy relacionada con la dislexia y el trastorno de déficit de atención (TDAH), en la población escolar entre el 3 y el $6 \%$ sufre de este trastorno de aprendizaje, Artigas-Pallarés (2002). Suyapan (2012) realizó un estudio para determinar las diferentes causas de las dificultades de aprendizaje que tiene la niñez, entre ellas está la dislexia que dificulta el español y la discalculia, que afecta la manera como se visualizan y se analizan los términos y conceptos matemáticos.

También es importante mencionar a estudiantes que no tienen discalculia, pueden presentar déficit de atención, hiperactividad o están quienes definitivamente no tienen afinidad con la materia o tienen desarrolladas otras clases de inteligencias y, por tanto, las matemáticas se les dificultan. El uso de videos como herramienta tecnológica les ayuda a concentrar la atención en determinado tema y a enfocarse en una sola cosa. Cabe decir que es importante que el diseño, ambientación, imágenes y sonido sean atractivos para el estudiantado (Burbules, 2012). 


\section{Procedimientos metodológicos}

El diseño empleado para el estudio fue el cuantitativo, para que el análisis de la información sea imparcial y más certera que con el uso de solo observación (Mendoza, 2006). "Por método cuantitativo los investigadores se refieren a las técnicas experimentales aleatorias, cuasi experimentales, test, análisis estadísticos multivariados, estudios de muestra, etc." (Cook y Reichard, 2005).

Las características por las cuales se seleccionó el modelo cuantitativo basado en el estudio de Mendoza (2006) son: permite examinar datos de manera numérica; ayuda a hacer demostraciones de cada variable separadas de su todo; se emplea una medición de resultados exhaustiva y detallada; se maneja la realidad desde una concepción objetiva, unitaria, estática y reduccionista y, finalmente, tiene la tendencia a servirse de los objetos de estudio, esto significa que el estudiantado es el principal participante del proceso.

El diseño de investigación experimental corresponde a la categoría de pretest, postest con un grupo (Rodríguez y Valldeoriola, 2008), ya que se emplearon dos test uno antes y otro después de la observación de los videos, para el estudio de los datos recolectados se establecieron tres categorías: los conceptos trabajados, el uso del video educativo y el proceso de enseñanza - aprendizaje.

La población seleccionada fueron estudiantes de un colegio privado de Bogotá -Colombia, el total de la población fue de 72 , la muestra que se tomó corresponde a 24 estudiantes de segundo grado, tienen en promedio 9 años, es un grupo heterogéneo, esto quiere decir que hay tres personas diagnosticadas con TDAH, una de las cuales pertenece al programa de aula alterna de la institución (proyecto de inclusión de niñez con dificultades cognitivas y físicas); dos estudiantes tienen dislexia y discalculia. El resto (19 estudiantes) no presenta dificultades de aprendizaje en matemáticas.

Además del estudiantado, se contó con un grupo de 25 docentes que trabajan en la institución, pero en diferentes áreas a la matemática y su trabajo no es específicamente con grado segundo.

Este tipo de muestra entra en la tipología de muestreo no aleatorio, ya que se toma un grupo completo de estudiantes, sin que sea voluntario o haya sido seleccionado al azar (Valenzuela y Flores, 2011). La muestra seleccionada representó a la población, ya que en el colegio de aplicación el estudiantado está repartido de forma equitativa en cada curso, esto quiere decir que en todas las condiciones académicas de estudiantes son similares y, por ende, es la muestra adecuada para realizar el estudio.

\section{Instrumentos}

Los instrumentos son el medio por el cual quien investiga recoge la información (Monje, 2011). Se seleccionaron los test de habilidades específicas (Anexo 1 y 2), en este caso las relacionadas con los algoritmos de la resta y a multiplicación por una cifra, estos test fueron estandarizados previamente; la observación (Anexo 4) y una encuesta realizada a estudiantes después del segundo test (Anexo 5).

\section{Test de habilidades específicas}

El test tiene como objeto recolectar información sobre características especiales de la personalidad, la conducta, entre otros; entre estos están los test de habilidades específicas, los cuales, empleando preguntas o actividades, son observadas y evaluadas por quien investiga (Aragón, et al, 2013). En este caso se emplearon test de habilidades específicas relacionadas con matemáticas.

Se emplearon los test para determinar cómo los niños desarrollaban el algoritmo de la resta y la multiplicación por una cifra. Se tomaron estos dos temas por ser los que mayor dificultad presentan. Cada test contó con 8 ejercicios, 4 por cada tema. 
Los dos test de habilidades, uno inicial o de diagnóstico (Anexo 1), y el otro para determinar los resultados del estudio (Anexo 2), tenían 4 restas y 4 multiplicaciones, dos de las restas eran reagrupando para números diferentes a cero y las otras con minuendos que tienen ceros y de las 4 multiplicaciones, dos eran sin llevar y dos llevando.

Los ejercicios propuestos fueron seleccionados de diferentes talleres y quices desarrollados por estudiantes de los otros dos grupos de segundo con anterioridad a la aplicación en el grupo muestra, las operaciones propuestas fueron las que presentaron un grado de dificultad mayor que las otras, razón por la cual se tomaron en cuenta para el estudio, de esta forma se realizó la estandarización de esta herramienta.

\section{La observación}

La observación es un registro sistémico, valido y confiable de comportamientos o actitudes que se muestran en un momento de estudio (Sampieri, Fernández y Baptista, 2006). Se aplica con dos propósitos: tener controladas las variables y establecer controles sobre los fenómenos estudiados (Monje, 2011).

La guía observación de comportamientos (Anexo 4) se realizó en el momento de ver los tres videos educativos ( Anexo 3). En la guía de observación se tenían en cuenta 4 aspectos, la atención, el gusto, la velocidad y la claridad del lenguaje. Se seleccionó la observación como complemento de las encuestas.

\section{La encuesta}

Es un instrumento que permite recolectar información mediante una cantidad no determinada de preguntas, puede tener preguntas cerradas, abiertas y precodificadas (González Y Calleja, 2009).

La encuesta (Anexo 5) se seleccionó por ser un elemento que proporciona las opiniones de los niños y esto da más veracidad al estudio. Esta contaba con siete preguntas, cinco (5) de respuesta cerrada y dos (2) de respuesta abierta. La encuesta a docentes (Anexo 6) fue realizada a profesorado de diferentes materias. Se creó empleando formularios de google drive y se envió por medio del correo electrónico.

\section{Procedimiento de recolección de datos}

Dentro del proceso de investigación, el test toma la etapa inicial, los videos afectan las competencias evaluadas y posteriormente se evidencia un cambio en el desarrollo de estos ejercicios, generando una reacción de causa - efecto, en la cual la causa fue observar los videos y el efecto es mejorar en la realización de ejercicios propios de la competencia matemática relativa a desarrollar algoritmos de resta y multiplicación.

El test inicial fue aplicado en el aula de clase, antes de la utilización de los videos, se evaluaron las competencias matemáticas de análisis y desarrollo de algoritmos en operaciones básicas y se reportaron los resultados. Posteriormente, se vio, con todo el grupo seleccionado, los videos correspondientes, con un televisor conectado a internet; luego de unos días se aplicó el segundo test en las mismas condiciones que el inicial, y se evaluaron las mismas competencias del test 1 y se recolectaron los datos de este en la misma forma de la inicial.

Por el tipo de investigación, la mejor herramienta para recolectar datos fue Microsoft Excel, se emplearon hojas de cálculo, se compararon y analizaron los datos obtenidos por estudiante, nivel y total. Cada dato recolectado fue transcrito en sistema binario para la organización de los resultados, el cero (0) representó una respuesta no favorable y el uno (1), una favorable. Se analizaron los datos tanto por estudiante, como por curso; para, desde los casos particulares, determinar conclusiones generales y con base en estos resultados generar a futuro un proyecto más amplio y completo. 


\section{ANÁLISIS Y DISCUSIÓN DE RESULTADOS}

En este apartado se presentarán los resultados según las categorías de análisis: conceptos trabajados y uso de videos educativos; y dentro de ellas se mostrarán los resultados de los instrumentos correspondientes a cada una. Se evidenciarán los resultados de las preguntas más relevantes y que proporcionaron respuestas a la pregunta de investigación.

\section{Conceptos trabajados}

Los conceptos que se trabajaron en este proyecto fueron el del algoritmo de la sustracción y de la multiplicación por una cifra en estudiantes de segundo primaria. Para evidenciar el manejo de estas competencias se aplicaron dos test, el pretest y otro después de observar los videos, el postest.

Para evaluar la competencia de solución de algoritmos para la resta, se tenían los cuatro ejercicios iniciales de cada test y para la de solución de algoritmos de la multiplicación se tenían los cuatro ejercicios finales.

En la Tabla 1 y la Figura 1 se comparan la cantidad de repuestas correctas (aciertos), de todas las preguntas tanto en el pretest, como en el postest, para los 24 niños del curso. Las preguntas de restas fueron denominadas como 1a, 1b, 1c y 1d y las de multiplicación como 2a, 2b, 2c y $2 \mathrm{~d}$.

Los primeros cuatro ejercicios eran de resta, la 1a era una sin reagrupación, el $1 \mathrm{~b}$ con reagrupación en una cifra, la 1c con reagrupación en una cifra que es cero (0) y la $1 \mathrm{~d}$ que correspondía a una resta con reagrupación en dos cifras que son ceros (0). En cuanto a los ejercicios de multiplicación se tenían el $2 \mathrm{a}$ y $2 \mathrm{~b}$ que correspondían a una sin llevar para sumar, la 2 c era llevando y la $2 \mathrm{~d}$ era hallar el producto de un número con cero y en la que había que llevar para sumar.

TABLA 1

Resultados de respuestas correctas de los dos test y porcentaje de aumento en respuestas acertadas

\begin{tabular}{|c|c|c|c|}
\hline \multicolumn{4}{|c|}{ Comparación de preguntas por test } \\
\hline Pregunta & Pretest & Postest & $\begin{array}{l}\text { Porcentaje } \\
\text { de } \\
\text { aumento }\end{array}$ \\
\hline la & 15 & 24 & 38 \\
\hline $1 \mathrm{~b}$ & 15 & 24 & 38 \\
\hline $1 \mathrm{c}$ & 18 & 23 & 21 \\
\hline $1 d$ & 17 & 23 & 25 \\
\hline $2 a$ & 22 & 24 & 8 \\
\hline $2 \mathrm{~b}$ & 21 & 24 & 13 \\
\hline $2 c$ & 10 & 21 & 46 \\
\hline $2 \mathrm{~d}$ & 11 & 20 & 38 \\
\hline
\end{tabular}




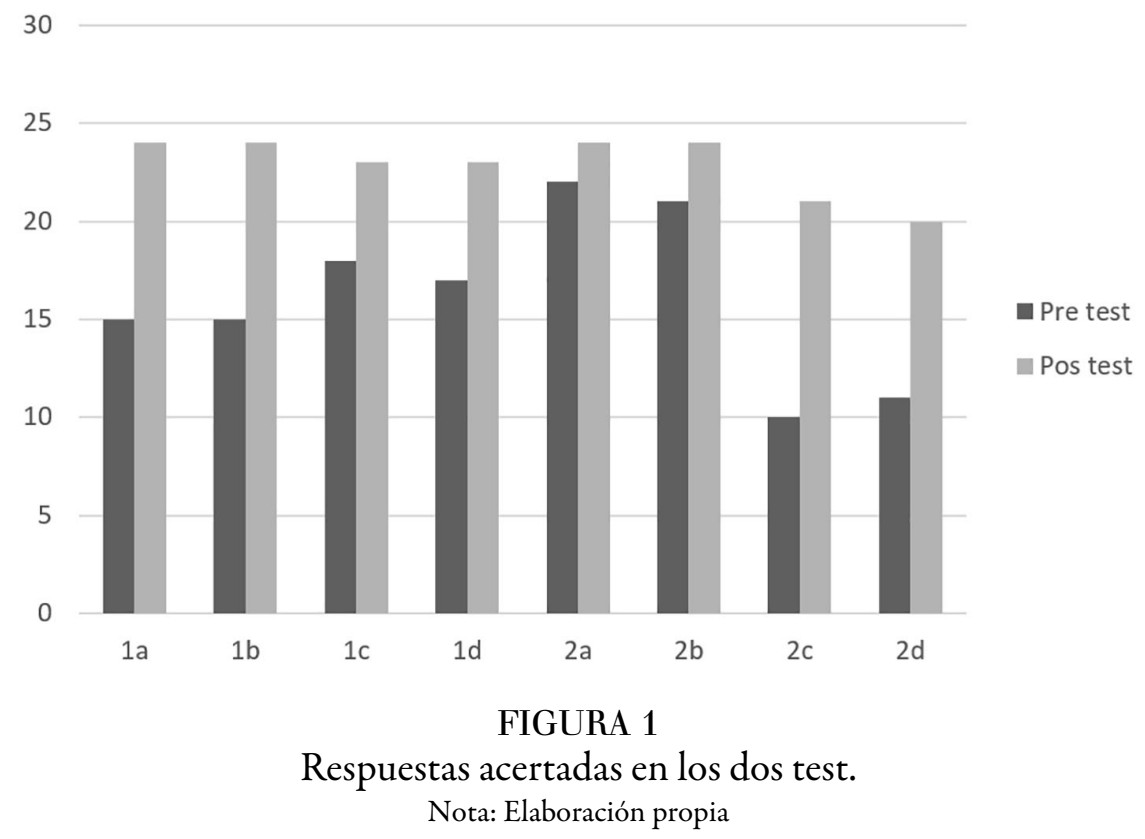

$\mathrm{Al}$ realizar el análisis de la Tabla 1 y la Figura 1, se puede observar un aumento entre el $8 \%$ y el 38 \%, en la cantidad de respuestas correctas, lo cual quiere decir que los niños mejoraron en su habilidad para resolver las operaciones planteadas. Las preguntas en las que más cambio se observo fue en la $1 \mathrm{~b}$, la $2 \mathrm{c}$ y la $2 \mathrm{~d}$., estas son las que más grado de dificultad presentan siempre, según la experiencia de aula.

Al realizar el análisis en cada uno de los grupos: los 5 estudiantes con dificultades de aprendizaje y los 19 que no las tienen, se obtienen la Tabla 2 y la Tabla 3, respectivamente.

La Tabla 2 y la Figura 2 muestran en comparación los resultados de 5 estudiante que presentan dificultades de aprendizaje, con TDAH y discalculia.

TABLA 2

Resultados estudiantes con dificultades

\begin{tabular}{llll}
\multicolumn{4}{l}{ Comparación preguntas estudiantes con dificultades } \\
Pregunta & Pretest & Postest & Porcentaje de aumento \\
1a & 2 & 5 & 60 \\
1b & 1 & 5 & 80 \\
1c & 2 & 4 & 40 \\
1d & 1 & 4 & 60 \\
2a & 3 & 5 & 40 \\
2b & 3 & 5 & 40 \\
2c & 0 & 5 & 100 \\
2d & 1 & 4 & 60
\end{tabular}




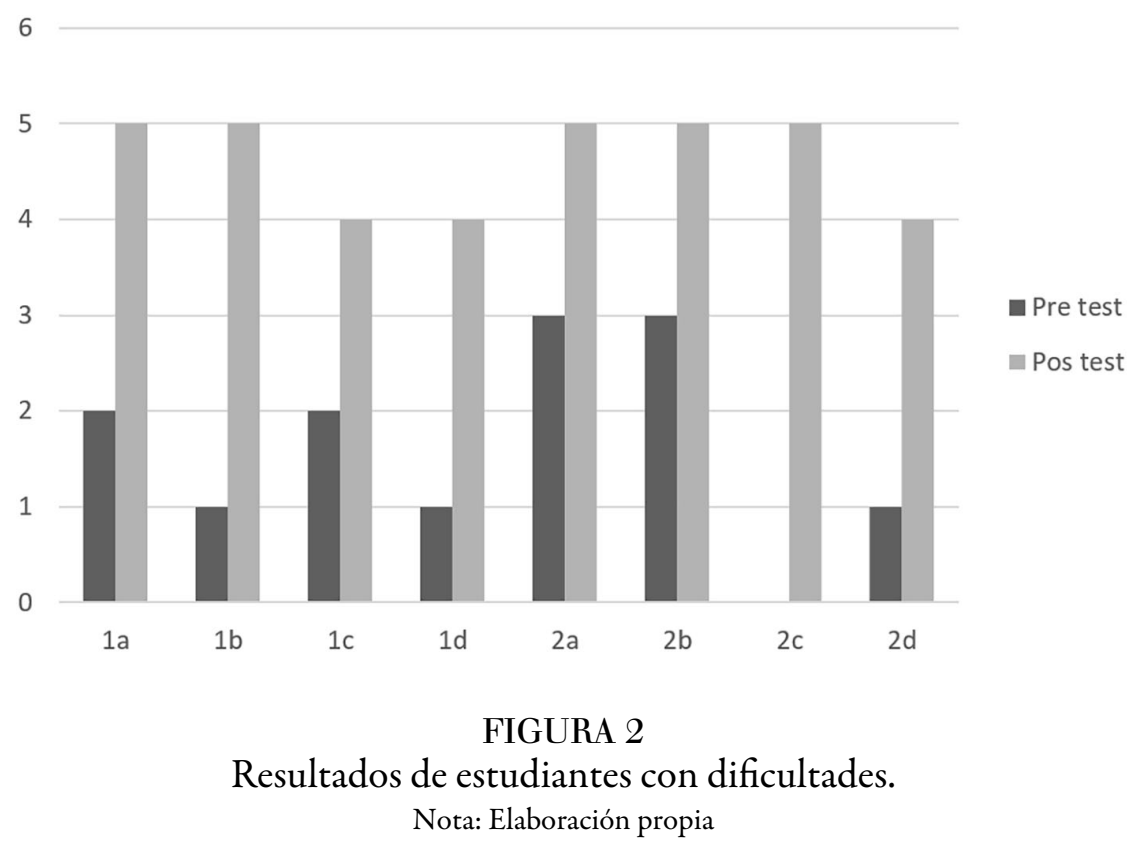

En la Tabla 2 y la Figura 2, se ve con claridad que en el grupo con dificultades es más notorio el cambio en sus resultados, en todas las preguntas se muestra una mejora significativa en especial en la 2c, en la cual pasó de nadie responderla de forma adecuada a hacerla de manera correcta en su totalidad.

En la Tabla 3 y la Figura 3, se muestran los resultados de los dos test en los 19 estudiantes que no presentan dificultades de aprendizaje.

TABLA 3

Comparación resultados estudiantes sin dificultades

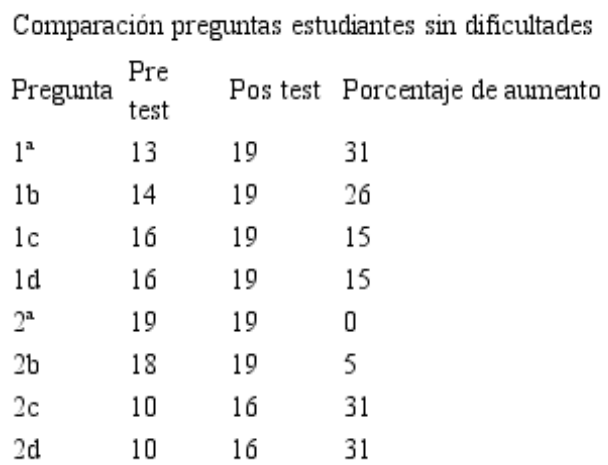




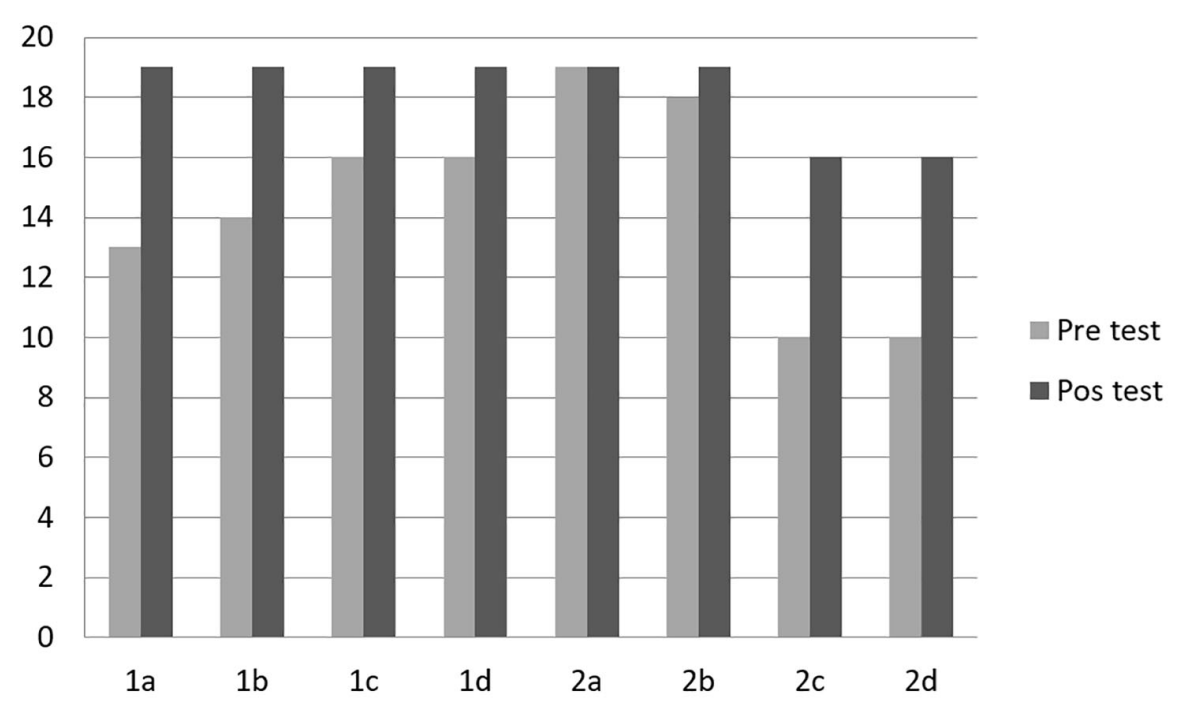

FIGURA 3

Resultados comparativos de estudiantes sin dificultades

Nota: Elaboración propia

\section{Uso del video educativo}

Para presentar los resultados de esta categoría es importante mostrar las respuestas obtenidas de la observación y algunas respuestas de las encuestas realizadas. Además, se deben conocer las características de cada video.

En los videos de resta se empleó un rectángulo formado por 100 cuadros pequeños, que representan la centena, una decena está representada por una barra con 10 cuadros, y las unidades son cuadros más pequeños. El primer video tenía una duración de 1:56 minutos, en este se muestra el proceso para realizar una resta en la cual se hace necesario descomponer las centenas y las decenas, en el video se representan el minuendo y cómo este se descompone para poder realizar la resta.

El segundo video tenía una duración de 2:10 minutos, este muestra una resta en la cual el minuendo tiene como cifras en las decenas y unidades el dígito cero, en el video se descompone una de las centenas en decenas y esa decena en unidades, el proceso se muestra simultáneamente con las imágenes y con las cifras numéricas, hasta tener el resultado de la resta. El tercer video tenía una duración de 1:50 y estaba dividido en dos partes, en ambas se muestra escribiendo paso a paso el proceso para multiplicar por una cifra, en la primera parte es una multiplicación en la cual no sobran decenas y en la segunda sí se deben sumar decenas después de multiplicar.

En la guía de observación se tenían en cuenta cuatro aspectos, dos califican a los niños y los otros los videos. El primero fue la atención, en la cual se tomaba nota sobre la atención que los grupos prestaron a los videos durante todo su tiempo de reproducción; otro criterio era el gusto, este se refería principalmente a la cara de agrado o desagrado que mostraban al verlos; la velocidad de los videos fue otra categoría, en esta se pretendía mirar, preguntando al terminar cada video si habían podido leer todos los textos, y se promediaron los resultados de los tres videos; por último se observó la claridad del lenguaje, preguntando si alguien no entendió alguna palabra al terminar los videos.

Los ítems que se tendrán en cuenta sobre la observación en este apartado son la atención y el gusto. En cuanto al primero se determinó que 20 del grupo de 24 mostraron mucha atención durante el tiempo 
empleado para la reproducción de los videos, esto equivale al $83 \%$ del curso. El segundo ítem, fue denominado gusto, en este 21 de 24 mostraron agrado por la presentación de los videos, esto equivale al 87\% (Tabla 4 ).

TABLA 4

Atención y gusto observados durante los videos

Si No Total Atención 20424

Gusto 21324

La tercera herramienta empleada fue la encuesta de estudiantes, la cual contaba con 7 preguntas. En la primera pregunta se hizo evidente que sintieron que los videos fueron de ayuda en su proceso, ya que el 100\% así lo respondió en su encuesta. En cuanto al gusto de los videos que se cuestionó en la segunda pregunta, todo el grupo determinó que alguno de ellos fue su favorito, el que más gustó fue el de multiplicaciones con un 52\%.

La tercera pregunta era: ¿Qué le cambiarías a los videos?, esta era una pregunta de respuesta abierta y por tal motivo las respuestas fueron agrupadas, entre las que más se repitieron, están; nada, esta la respondieron 14 estudiantes, que corresponde al $58 \%$, la mano que escribe, fue la respuesta de 3 , correspondiente al $13 \%$, entre las otras 7 respuestas, que son el 29\%, se tienen: "todo menos los ositos", "explicar cómo se usan las regletas", "cambiar la música por alguien que explique", entre otras (Tabla 5).

TABLA 5

Respuestas de “¿Qué le cambiarías a los videos?

Respuestas frecuentes Pregunta 3

Nada 14 La mano 3 Otras 7 Total 24

La cuarta pregunta era: “¿Te sentiste con más seguridad al hacer el postest después de ver los videos?”, la cual tenía respuesta cerrada y las opciones de respuesta era "sí" o "no", 22 estudiantes respondieron "sí", equivalentes al $92 \%$ y 2 que "no" (Tabla 6 ).

TABLA 6

Respuestas de “¿Te sentiste más seguro al hacer el post test después de ver los videos?

Pregunta 4 Si 22 No 2 Total 24

La quinta pregunta fue: ¿Cuántas veces viste los videos? (Tabla 7), las opciones de respuesta eran 1, 2, 3, 4 o más de 4 . La primera respuesta fue la seleccionada por 16 estudiantes, esto equivale al $84 \%$; la segunda respuesta obtuvo $0 \%$; la tercera respuesta 1 , equivalente al $5 \%$, la cuarta respuesta no tuvo frecuencia y la quinta dos estudiantes, esto es el $11 \%$.

TABLA 7

Respuesta de “¿Cuántas veces viste el video?”

Pregunta 511620314

0 Más de 42 Total 19

En la Tabla 8, se muestra la cantidad de veces que estudiantes con dificultades vieron los videos, en esta E1, E2 y E3, hacen referencia a estudiantes con TDAH y E4 y E5 con discalculia. 
TABLA 8

Número de veces que estudiantes con dificultades vieron los videos

$\begin{array}{ll}\text { Estudiante } & \text { Cantidad } \\ \text { E1 } & 2 \\ \text { E2 } & 3 \\ \text { E3 } & 3 \\ \text { E4 } & 2 \\ \text { E5 } & 1\end{array}$

\section{Análisis de resultados}

El análisis se realizó teniendo en cuenta las categorías de estudio, las cuales eran: conceptos trabajados, uso de videos educativos y enseñanza- aprendizaje, estas categorías ayudan a dar repuesta a la pregunta de investigación: ¿Qué efectos se evidencian al implementar videos educativos como herramienta disruptiva para apoyar el proceso de aprendizaje de las matemáticas en los niños de educación básica primaria?

La primera categoría de estudio fue la de conceptos trabajados, en este proyecto, el algoritmo de la sustracción y de la multiplicación, en los ocho (8) se evidenció un aumento en la capacidad del grupo de estudiantes para solucionar los algoritmos después de interactuar con los videos. Esto se puede asegurar a través de los ejercicios trabajados en los test de habilidades específicas donde se observa el aumento de respuestas acertadas compiladas en la tabla 1 , que fue entre el $8 \%$ y el $48 \%$, y en las respuestas obtenidas en la encuesta donde expresaron sentir mayor seguridad al enfrentar el segundo test, después de ver los videos. El estudiantado debe dominar procesos y algoritmos matemáticos al igual que saber cuándo usarlos, para, de esta manera, unir un acto procedimental con un concepto (Matemáticas, 2007). El hecho de que mejoren en su habilidad para solucionar algoritmos empleando videos educativos, ayuda a encontrar un camino para garantizar la unión entre proceso y concepto matemático.

Analizando los resultados de estudiantes con dificultades y en general, y la cantidad de veces que vieron los videos, se puede determinar que, a mayor cantidad de veces observados, mayor es la posibilidad de acierto y de seguridad al realizar la tarea propuesta. Esto se hace evidente en la comparación de las respuestas de los test que se encuentran en la tabla 2, en la cual se identifica un aumento entre el $40 \%$ y el $100 \%$ en uno de los ejercicios. Greenberg y Zanetis, (2012) establecen que los videos ayudan a consolidar conocimientos gracias a los estímulos audiovisuales y auditivos que contienen. Los videos aumentaron la competencia de estos niños para desarrollar algoritmos de resta y multiplicación, el estudiantado que no mejoró en ningún ejercicio corresponden a quienes manifestaron solo haber visto los videos en el aula.

En la segunda categoría: uso de videos educativos, se evidenció un aumento en los tiempos de atención de estudiantes, ya que les gustaron las imágenes presentadas en los videos. Esto se evidencia en el $83 \%$ que se observaron con atención y de manera concentrada durante la reproducción. Según Burbules (2012), es importante que el diseño, ambientación, imágenes y sonidos sean atractivos para el estudiantado, ya que esto ayuda a crear ambientes diferentes de aprendizaje y motivan el cerebro para afianzar el conocimiento (Sousa, 2006). La atención es una herramienta que permite al cerebro conectar las imágenes vistas con el conocimiento matemático que se trabajó en el proyecto.

Estudiantes con discalculia y con TDAH que vieron los videos mayor número de veces realizaron de forma correcta todos los ejercicios del segundo test, el niño que solo lo vio una vez fue quien presentó falencias. Esto se evidencia al realizar el contraste entre la Tabla 2 y la Tabla 7, y observar los resultados de aumento versus la cantidad de veces que se vieron los videos. Según Sousa (2006), cuanto más valor se le puedan dar a las experiencias vividas, se puede garantizar una mayor apropiación del conocimiento. El estudiantado que 
sintió entender los algoritmos al ver los videos, lo repitieron más veces con el fin de sentir mayor seguridad en sus conocimientos.

\section{Conclusiones}

Una de las conclusiones de este trabajo es que el uso de los videos produjo beneficios al estudiantado con dislexia y TDAH, ya que en los ocho ejercicios propuestos presentaron mejores resultados, quienes tenían algún tipo de dificultad de aprendizaje mostraron más atención y se centraron al ver los videos y desarrollar el postest. Esto muestra que los videos ayudan a superar dificultades de aprendizaje y otorgan más seguridad frente a nuevas tareas.

En cuanto al grupo que no presenta dificultades de aprendizaje se evidenció que los empleó como fuente de consulta y estudio, lo cual le ayudó a mejorar sus resultados o a afianzar conocimientos.

Otro efecto fue que el estudiantado se mostró con mayor seguridad al desarrollar el segundo test, el cual fue contestado después de ver los videos, esto se hizo notorio en los resultados de los ejercicios. Para los niños con alguna dificultad de aprendizaje, el poder ver los videos a su propio ritmo garantizó un mayor afianzamiento de los conceptos trabajados.

Quienes vieron los videos en repetidas ocasiones y tenían dificultades mejoraron notablemente en el segundo test, esto quedó demostrado en la recolección y comparación de datos de los dos test y finamente se puede determinar que hubo una disminución de la brecha conceptual entre niños con dificultades y sus pares.

Por lo anterior, se puede concluir que el proceso de enseñanza - aprendizaje se ve altamente beneficiado con el uso de herramientas disruptivas como los videos educativos, además aumenta el interés por aprender matemáticas, esto se refleja en el incremento del $8 \%$ al $48 \%$ en adquisición de conceptos de sustracción y multiplicación.

\section{RECOMENDACIONES}

Una dificultad que se presentó al realizar el proyecto fue encontrar el espacio para que el grupo pudiera ver los videos, y hacer la observación de estudiantes al mismo tiempo, por tanto, es recomendable que para este tipo de proyectos, en los cuales se involucra la tecnología en función del aprendizaje, las instituciones cuenten realmente con todos los espacios y herramientas necesarios.

En cuanto a la población, sería interesante trabajar este proyecto con un grupo infantil que en su totalidad presente dificultades de aprendizaje, y hacer un seguimiento por casos, para dar más riqueza institucional al trabajo de inclusión que se realiza en esta misma, con el aula alterna.

A nivel institucional, se debe motivar y capacitar al personal docente en la elaboración de videos educativos propios que den riqueza al modelo educativo de la institución y no sean empleados solamente en los grados de primaria, este proyecto debe ser implementado en todos los niveles educativos, con el fin de apoyar el proceso de aprendizaje en especial de los niños con dificultes para la matemática.

\section{SUgERENCIAS PARA ESTUDIOS FUTUROS}

Después de realizar el proyecto surgen algunas preguntas, tales como: ¿Qué ocurre cuando estos videos se emplean en una población con dificultades de aprendizaje? ¿Qué herramientas necesitan los maestros y maestras para crear videos educativos? Las preguntas anteriores pueden dar paso a proyectos interdisciplinarios y trasversales que fortalezcan los procesos de enseñanza -a aprendizaje de cualquier institución. 
En cuanto a preguntas que permitan mejorar el presente proyecto estarían: ¿Qué tan duraderos son los conceptos trabajados con videos en contraste con los temas estudiados de forma tradicional sin uso de tecnología?, ya que en este estudio no se determinó, si tiempo después de la visualización de los videos, los niños y las niñas conservaban todavía el proceso algorítmico estudiado.

Este proyecto es solo el inicio de un gran trabajo que se va realizar a nivel institucional, en el cual se tendrán en cuenta las dificultades estudiantiles, sus gustos y su forma de aprender, para generar videos, que ayuden al personal docente a potenciar todas las habilidades del pensamiento y crear, de esta forma, una sociedad con una gran mentalidad de cambio y muchas ganas de seguir aprendiendo toda la vida.

De la observación de los niños se tomaron el tercer y cuarto ítem, en el tercer ítem se establecía si la velocidad de reproducción era adecuada, 19 de 24 estudiantes dijeron que sí pudieron leer y entender los textos presentes en los videos, esto equivale al 79\% del curso. En el cuarto ítem en el cual se establecía si la claridad del lenguaje empleado en los videos, 22 de 24 manifestaron que el lenguaje era claro y lo entendieron bien. Esto equivale al $62 \%$ del curso.

\section{REFERENCIAS}

Alfaro, G. S., Monroy, N., y Pinzón, J. L. L. (2013). Cálculo diferencial: Aprendiendo con nuevas tecnologías. Revista de Tecnología, 12(2), 42-51.

Aranda, M. Pérez, I. Sánchez, B. (2009). Dificultades en el aprendizaje matemático. Recuperado de https://www.uam.es/personal_pdi/stmaria/resteban/Archivo/TrabajosDeClase/ DificultadesMatematicasLenguaje1.pdf

Aragón, E. L., Delgado, C. I., Aguilar, M., Araújo, A. y Navarro, J. I. (2013). Estudio de la influencia de la inteligencia y el género en la evaluación matemática temprana. European journal of education and psychology, 6(1), 5-18.

Artigas-Pallarés, J. (2002). Problemas asociados a la dislexia. Revista de Neurología, 34(11), 7-13. Recuperado de http://ardilladigital.com/DOCUMENTOS/EDUCACION\%20ESPECIAL/ LOGOPEDIA/TRASTORNOS\%20LENGUAJE/DISLEXIAS/Problemas\%20asociados\%20a\%20la \%20dislexia\%20-\%20Artigas\%20-\%20art.pdf

Boude, O. (2013). Tecnologías emergentes en la educación: Una experiencia de formación de docentes que fomenta el diseño de ambientes de aprendizaje. Educação \& Sociedade, 34(123), 531-548. Centro de Estudos Educação e Socieda de Campinas. Brasil. Recuperado de http://www.redalyc.org/articulo.oa?id=87328002014

Burbules, N. (2012). El aprendizaje ubicuo y el futuro de la enseñanza. Encuentros de Educación. 13, 3-14. University of IIIinois- Champaign. Estados Unidos. Recuperado de http://library.queensu.ca/ojs/index.php/encounters/ article/viewFile/4472/4498

Carneiro, R., Toscano, J., y Díaz, T. (Coords.). (2009). Los desafíos de las TIC para el cambio educativo. Colección Metas Educativas. OEI/Fundación Santillana. Madrid. España. Recuperado de http://www.oei.es/metas2021/ LASTIC2.pdf

Cobo, J. (2009). El concepto de tecnologías de la información. Benchmarking sobre las definiciones de las TIC en la sociedad del conocimiento. Revista Zer, 14(27). Recuperado de: http://www.ehu.eus/zer/hemeroteca/pdfs/ zer27-14-cobo.pdf

Cook, T.D, Reichard, CH. (2005). Métodos cualitativos y cuantitativos en investigación educativa. Ediciones Morata. Madrid.

González, A., Calleja, V., (2009). Los estudios de encuesta. Métodos de investigación en educación. UAM. Recuperado de https://www.uam.es/personal_pdi/stmaria/jmurillo/InvestigacionEE/Presentaciones/Encuesta_doc.pdf

Greenberg, A., Zanetis, J. (2012). The impact of Broadcast and Streaming Video in Education. Report commissioned by Cisco Systems Inc. to Wainhouse Research, LLC. San Jose, CA. USA. Recuperado de http://www.cisco.com/ web/strategy/docs/education/ciscovideowp.pdf 
Ley N.o 1341 del 30 de Julio del 2009 de la Republica de Colombia. Recuperado de http://www.alcaldiabogota.gov.co/ sisjur/normas/Normal.jpp?i=36913

Llinares, S. (2004). La generación y uso de instrumentos para la práctica de enseñar matemáticas en educación primaria. España. Recuperado de http://dipmat.math.unipa.it/ grim/dott_HD_MphCh/Llinares_6_04_Esp.pdf

Mendoza, R. (2006). Investigación cualitativa y cuantitativa, diferencias y limitaciones. Monografía. Perú.

Monje, C. (2011). Metodología de la investigación cuantitativa y cualitativa. Universidad Surcolombiana. Recuperado de https://carmonje.wikispaces.com/file/view/Monje+Carlos+Arturo+-+Gu\%C3\%ADa+did\%C3\%Alctica + Metodolog\%C3\%ADa+de+la+investigaci\%C3\%B3n.pdf

Ministerio de Cultura. (2014). Proyecto Uso y apropiación de TIC. Biblioteca nacional de Colombia. Recuperado de http://www.bibliotecanacional.gov.co/content/proyecto-uso-y-apropiaci\%C3\%B3n-de-tic

Peña, O, García, R. y Ramírez, M. S. (2010). La selección del énfasis en el programa de gerontología a través del uso de videos en el proceso enseñanza- aprendizaje. Palabra educativa, 1, 69-79. Recuperado de http:// catedra.ruv.itesm.mx/handle/987654321/746

Rodríguez, D., Valldeoriola, J. (2008). Metodología de la investigación. Universidad Oberta de cataluya. Recuperado de http://www.zanadoria.com/syllabi/m1018/mat_cast/PID_00148543-1.pdf

Rodríguez, S. (14 de febrero, 2011), Khan Academy: Como en el cole. El mundo. Recuperado de http:// www.elmundo.es/blogs/elmundo/catalejo/2011/02/14/khan-academy-como-en-el-cole.html

Rosselli, M. y Matute, E. (2011). La neuropsicología del desarrollo típico y atípico de las habilidades numéricas. Revista Neuropsicología, Neuropsiquiatría y Neurociencias, 11(1), 123-140. Recuperado de http:// neurociencias.udea.edu.co/revista/PDF/REVNEURO_vol11_num1_12.pdf

Rubio, A. (2010). Jóvenes en red, generación digital y cambio social. XXVIII Jornades de Polítiques Locals de Joventut. Madrid. Recuperado de http://www.diba.cat/documents/95670/96986/joventut-fitxers-2010rubio-pdf.pdf

Sampieri, R., Fernández, C., Baptista, P. (2006). Metodología de la investigación (4ta edic.). México D.F.: Editorial Mc Graw Hill.

Serra, J. (2015). La discalculia. Recuperado de http //discalculia.es/discalculia/Inicio.html

Sousa, D. (2006). How the brain learns (3rd. edition). Editorial Corwin Press.

Suyapan, M. (2012). Investigación educativa en niños y niñas con dificultades específicas de aprendizaje relacionada con el conocimiento y disposición en los docentes de las escuelas de aplicación del departamento de Comayagua (Tesis de maestría). Recuperado de http://www.upnfm.edu.hn/bibliod/images/stories/tesnov/ Formaciondeformadores/Marlen\%20Suyapa\%20Izaguirre\%20Mejia.pdf

Valenzuela, J. R. y Flores, M. (2011). Fundamentos de investigación educativa [Recurso electrónico]. (Vol. 2: El proceso de investigación educativa). Monterrey, México: Editorial Digital del Tecnológico de Monterrey. 
Anexo 1

Test 1

Nombre: Curso:

1. Soluciona las siguientes restas:

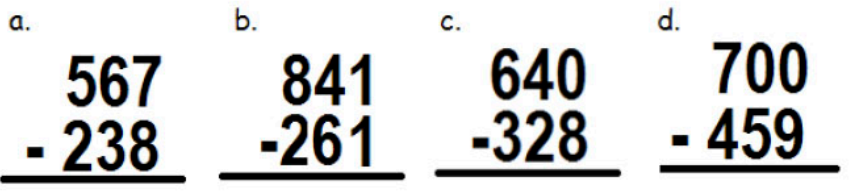

2. Soluciona las siguientes multiplicaciones

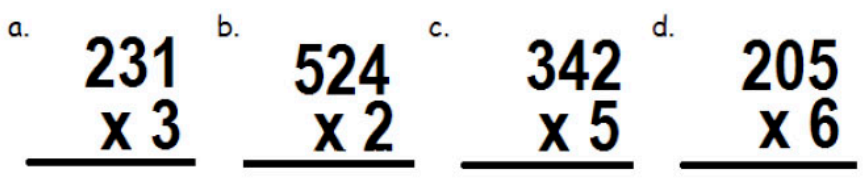

ANEXO 1

Test inicial

Anexo 2

Test 2

Nombre: Curso:

1. Soluciona las siguientes restas:

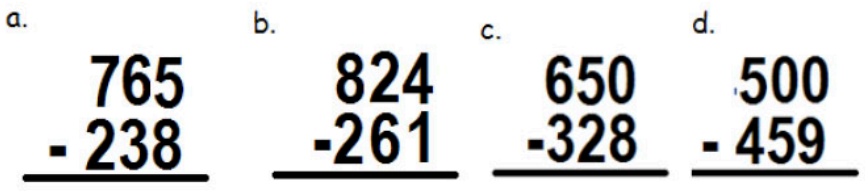

2. Soluciona las siguientes multiplicaciones \begin{tabular}{rrr}
331 & 724 & 543 \\
$\times 2$ & 305 \\
$\times 2$ & $\times 5$ \\
\hline
\end{tabular}

ANEXO 2

Test 2 o post test 
Angélica María Velasco Guardias, et al. Los videos educativos como herramienta disRuptiva Para apo...

\section{Anexo 3}

Este es el video para realizar restas descomponiendo.

El link del video es: https://www.youtube.com/ watch? $=\mathrm{HXgBJhNt} 1 \mathrm{ww} \& \mathrm{rel}=0$
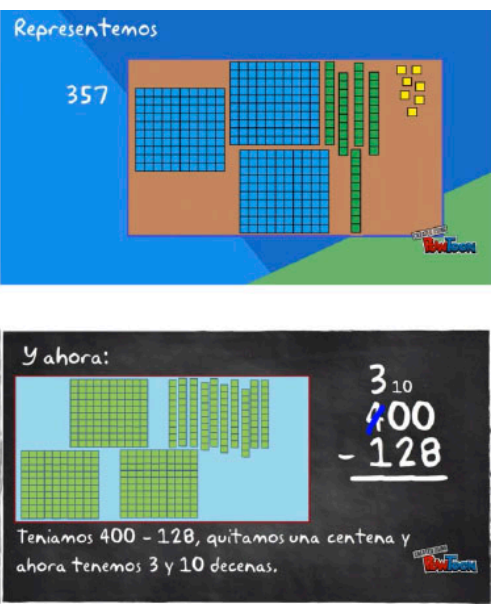

Este video es para estudiar las restas con números que tengan ceros.

El link es el siguiente: https://www.youtube.com/ watch?v=qn38quJnjtY\&rel=0

Este video es para estudiar el algoritmo de la multiplicación. El link es: https://www.youtube.com/watch?v=fPz vlVzeZk\&rel $=0$

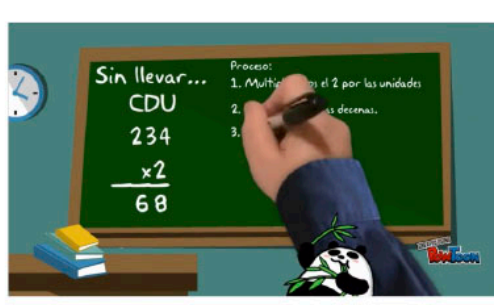

ANEXO 3

Links de los videos y pantallazos

\section{Anexo 4}

\begin{tabular}{|l|l|l|l|}
\hline \multicolumn{1}{|c|}{ Criterio } & Sí & No & Total \\
\hline Atención: miran atentamente los videos & & & \\
\hline Gusto: ven con agrado los videos & & & \\
\hline Velocidad de los videos: pueden leer los textos completos & & & \\
\hline Claridad del lenguaje: preguntan algunos términos & & & \\
\hline
\end{tabular}

ANEXO 4

Tabla de observación 


\section{Anexo 5}

Nombre:

1. ¿Crees que estos videos te ayudan para aprender o repasar?

$\square$ sí $\square \mathrm{NO}$

2. ¿Cuál fue el video que más te gustó?

El de multiplicaciones

El de restas llevando

El de restas con números 1que tengan ceros

Todos

Ninguno. Si esta es tu respuesta. Por favor escribe por qué

3. ¿Qué le cambiarías a los videos?

4. ¿Te sentiste más seguro al hacer el test 2 después de ver los videos?

$\square$ SI $\square$ NO

5. ¿Cuántas veces viste los videos?

$\square 1$

$-2$

3

Más de 4

6. ¿En qué otras materias empleas videos para estudiar?

7. De 1 a 10 qué calificación le das a cada video

El de multiplicaciones

El de restas llevando

El de restas con números 1que tengan ceros

ANEXO 5

Encuesta a estudiantes

CC BY-NC-ND 